Research Article

\title{
Solution of Fractional Partial Differential Equations Using Fractional Power Series Method
}

\author{
Asif Iqbal Ali (iD, Muhammad Kalim, and Adnan Khan (iD \\ Department of Mathematics, National College of Business Administration \& Economics (NCBA \& E), Lahore, Punjab, Pakistan \\ Correspondence should be addressed to Asif Iqbal Ali; asifiqbalali@ncbae.edu.pk
}

Received 28 May 2021; Revised 26 September 2021; Accepted 16 October 2021; Published 5 November 2021

Academic Editor: Elena Braverman

Copyright (C) 2021 Asif Iqbal Ali et al. This is an open access article distributed under the Creative Commons Attribution License, which permits unrestricted use, distribution, and reproduction in any medium, provided the original work is properly cited.

In this paper, we are presenting our work where the noninteger order partial differential equation is studied analytically and numerically using the noninteger power series technique, proposed to solve a noninteger differential equation. We are familiar with a coupled system of the nonlinear partial differential equation (NLPDE). Noninteger derivatives are considered in the Caputo operator. The fractional-order power series technique for finding the nonlinear fractional-order partial differential equation is found to be relatively simple in implementation with an application of the direct power series method. We obtained the solution of nonlinear dispersive equations which are used in electromagnetic and optics signal transformation. The proposed approach of using the noninteger power series technique appears to have a good chance of lowering the computational cost of solving such problems significantly. How to paradigm an initial representation plays an important role in the subsequent process, and a few examples are provided to clarify the initial solution collection.

\section{Introduction}

Mathematical equations containing two or more independent variables are called partial differential equations (PDEs). The highest derivative in the PDEs is called the order of the equation. The specific solution of the PDEs is a function that solves the equation, which can be verified by substituting the solution into the equation and obtaining an identity. The solution containing all the particular solutions of the equation in question is called the general solution. The term used to express a specific solution for second-order and higher-order nonlinear partial differential equations is called an exact solution. Problems involving the function of many variables, such as heat or sound propagation, static electricity, electrodynamics, and fluid flow, are solved by PDE $[1,2]$.

Generally, the solution with unknown coefficients is assumed to be a power series, and then the solution is substituted into the differential equation to find the recurrence relationship of the coefficients. New applications in fluid dynamics, viscoelasticity, mathematical biology, electrochemistry, and physics have led to the latest development in fractional DE. Using data from experiments, fractional DEs have recently proved to be useful tools for simulating many physical phenomena and fractional PDEs have been suggested for filtering flows in porous media. The examples that have been studied and solved include the space-time fractional diffusion wave equation, fractional advection scattering equation, fractional telegraph equation, fractional $\mathrm{KdV}$ equation, and linear nonhomogeneous fractional partial differential equations $[2,3]$.

Nowadays, the uses of nonlinear partial (or ordinary) differential equations in the practice of multidisciplinary research are considered to be an important technique for solving real-life problems that have social and public-health implications. Researchers in different scientific areas like biology, physiology, cell biology, chemistry, chemical physics, engineering, and systematic natural sciences are including applied mathematical methods in their practices. These applications are not only useful for technological advances in computer science to study cancers, hereditary diseases, tumor formation, kinematic processes, and DNA communication processes but also in mathematical controls to supply a successful cancer therapy. Indeed, there are many 
biomedical engineering applications for studying biological problems involving highly nonlinear and sophisticated collaborative processes, where the most promising theory available to conduct such studies is complex dynamical systems theory. The description of this theory almost entirely depends on nonlinear partial differential equations (NLPDE). Power series solution (PSS) technique has been restricted to unravel the linear differential equations, i.e., ordinary differential equations (ODEs) $[1,2]$ and partial differential equations (PDEs) $[4,5]$.

Linear partial differential equations have traditionally been overcome using the variable separation method because it creates an ODE system that is easier to decipher with PSSM. Examples of them are the spherical harmonics used and the Legendre polynomials in the Bessel equation in cylindrical coordinates or the Laplace equation in spherical coordinates $[4,5]$. It is understood that in NLPDE, as we remember, variable separation technology cannot be used. The way to unlock NLPDE is to use approximate analysis techniques to get the answer, that is, semianalytical or nondigital, direct or indirect. Directly, there are methods such as loose operator formalism [6] or inverse dispersion transformation [7]. In a direct way, for instance, the PSS can be used in an asymptotic calculation of the Hirota technique linking a bilinear operator method [8] and the homotopy analysis method (HAM) $[9,10]$ and the Adomian decomposition technique [11]. The latest approach requires a sequence of expansions with a nonsmall perturbation parameter estimate to change the convergence. This approach is distinct from the standard perturbation theory. Methods, also simpler, to estimate the representation in NLPDE are the Taylor polynomial approximation (TPA) technique $[12,13]$ and therefore for the power series solution method (PSSM). In each technique, a semianalytical representation is attained to implement the PSS technique. However, no use has been made of the PSSM to decipher the nonlinear ODE [14-17] or NLPDE [18-20]. Because NLPDEs do not have exact solutions in general, it is hard to define the most efficient method to be used; indeed, the only way to establish the efficiencies of the relevant methods involves employing experimental methods.

Further information on how to solve NLPDEs by using a point contact, Bäcklund, hodograph, Legendre, or Euler transformations or by applying Lie algebras and groups can be found in reference [21]. There is an immense work on noninteger calculus, and this has been grown exponentially in recent years, with noninteger ODEs becoming commonly used as mathematical prototypes [22, 23]. Recent studies consist of the solution of fractal media [24], viscoelastic materials [23], economics and finance [22, 25], compartment models [26], in porous media [27], epidemiology [28], nerve cell signaling [29], and anomalous diffusion system [30]. From a modeling perspective, the interpretation of the representation as an algebraic countenance involving functions of structure strictures is commonly pursued. Important progress has been made concerning linear noninteger ODEs by expanding proven techniques of elucidation for linear nonfractional ODEs. The Laplace transform technique can be extended to noninteger linear ODEs, with constant coefficients (see [31-33]).

The disadvantage of this method depends on the complexity of the Laplace transform of such functions, regardless of whether the equations are homogeneous or not, or by inverting the Laplace transform based on a calibrated computable function. In the noninteger linear ODE, with variable coefficients, the Laplace transform method has an additional limitation, that is, the Laplace transform of the product of the function is only available in special cases. In nonfractional regular linear ODEs, with different coefficients, the series expansion method is considered to help clarify the problem. Examples of power series can be seen in [34-48]. The description of this theory is also related to the use of the residual power series method [49] to solve spacetime fractional PDEs. The comparison of Aboodh transformation and differential transformation method (DTM) numerically in result solution obtained is compared the solution by DTM is rapid convergent [50]. Several papers have been dedicated to the application of series expansion approaches to linear noninteger ODEs which basically is the subject of this article.

\section{Fundamental Concepts}

Definition 1. The Caputo noninteger derivative operator of order $\mu$ with respect to " $t$ " is defined in the subsequent arrangement.

$$
\begin{aligned}
& \mathbf{D}_{\mathbf{t}}^{\mu} \mathbf{h}(\mathbf{u})=\mathbf{D}^{\mu} \mathbf{h}(\mathbf{u})=\mathbf{J}^{\mathbf{n}-\mu} \mathbf{D}^{\mathbf{n}} \mathbf{h}(\mathbf{u}), \\
& D^{\mu} h(u)=(\Gamma(n-\mu))^{-1} \int_{0}^{u}(u-t)^{-\mu+n-1} h^{(n)}(t) \mathrm{d} t,
\end{aligned}
$$

where $\mu>0, u>0, n \geq \mu>n-1, n \in \mathbb{N}$.

In a comparable manner of nonfractional-order differentiation, the Caputo noninteger derivative operator is a linear operation.

$$
D^{\mu}(\alpha f(u)+\beta g(u))=\alpha D^{\mu} f(u)+\beta D^{\mu} g(u),
$$

where $\alpha$ and $\beta$ are constants. We have $D^{\mu} k=0$ for the Caputo's derivative, if $k$ is constant.

$$
D^{\mu} u^{m}= \begin{cases}0, & \text { for } m \in \mathbb{N}_{0} \text { and } m<\mu, \\ \frac{\Gamma(m+1)}{\Gamma(m-\mu+1)} u^{m-\mu}, & \text { for } m \in \mathbb{N}_{0} \text { and } m \geq \mu .\end{cases}
$$

We practice the maximum function $\mu$ to signify the least integer larger than or equivalent to $\mu$, and $\mathbb{N}_{0}=\{0,1,2, \ldots\}$. Evoke that for $\mu \in \mathbb{N}$, the Caputo differential operator corresponds with the normal differential operator of nonfractional order.

Definition 2. For the variable $u$ and coefficients $a_{n}(n=0,1, \ldots, \infty)$, if $u>u_{0}$, the fractional power series (FPS) about the point $u_{0}$ is defined as 


$$
\begin{aligned}
\sum_{n=0}^{\infty} a_{n}\left(u-u_{0}\right)^{n \mu}= & a_{0}+\left(u-u_{0}\right)^{\mu} a_{1}+\left(u-u_{0}\right)^{2 \mu} a_{2} \\
& +\left(u-u_{0}\right)^{3 \mu} a_{3}+\cdots,
\end{aligned}
$$

where $\mu>0, m \geq \mu>m-1, m \in \mathbb{N}^{+}$.

Theorem 1. Let the radius of convergence for the function with fractional power series (FPS) representation $h(u)=\sum_{n=0}^{\infty} a_{n} u^{n \mu}, 0 \leq u<R$, be greater than zero (i.e., $R>0$ ).

Then, for $m \in \mathbb{N}^{+}$and $m-1<\mu \leq m$, the following expression holds true:

$$
D^{\mu}(h(u))=\sum_{m=1}^{\infty} a_{m} \frac{\Gamma(m \mu+1) u^{(m-1) \mu}}{\Gamma((m-1) \mu+1)} .
$$

Proof. From the linearity concept of Caputo derivative and the idea of power series derivative, we have

$$
\begin{aligned}
D^{\mu}(h(u)) & =D^{\mu} \sum_{m=0}^{\infty} a_{m} u^{m \mu}=D^{\mu}\left(a_{0}+a_{1} u^{\mu}+a_{2} u^{2 \mu}+\cdots\right) \\
& =D^{\mu} a_{0}+a_{1} D^{\mu} u^{\mu}+a_{2} D^{\mu} u^{2 \mu}+\cdots \\
& =a_{1} D^{\mu} u^{\mu}+a_{2} D^{\mu} u^{2 \mu}+\cdots \\
D^{\mu}(h(u)) & =\sum_{m=1}^{\infty} a_{m} D^{\mu} u^{m \mu} .
\end{aligned}
$$

From the power rule of the Caputo derivative, we have

$$
D^{\mu} u^{m \mu}= \begin{cases}0, & \text { for } m \mu \in \mathbb{N}_{0} \text { and } m \mu<\mu, \\ \frac{\Gamma(m \mu+1)}{\Gamma((m-1) \mu+1)} u^{(m-1) \mu}, & \text { for } m \mu \in \mathbb{N}_{0} \text { and } m \mu \geq \mu, \\ \mathbb{N}_{0}=\{0,1,2, \ldots\}, \mu \in \mathbb{Z}, \mu \geq \mu .\end{cases}
$$

From equations (3) and (5), we arrive at

$$
D^{\mu}(h(u))=\sum_{m=1}^{\infty} a_{m} D^{\mu} u^{m \mu}=\sum_{m=1}^{\infty} a_{m} \frac{\Gamma(m \mu+1)}{\Gamma((m-1) \mu+1)} u^{(m-1) \mu},
$$

where $h(u)=\sum_{m=0}^{\infty} a_{m} u^{m u}$.

2.1. Application. In order to validate the high degree of efficiency and precision of the projected FPS approach for unraveling fractional-order systems, numerical forms and instances are pragmatic. The reader can discover a sketch and applications for this technique in [42]. Computations were accomplished by using MATLAB. Examples are taken from [32].
Example 1. Consider the succeeding noninteger order structure:

$$
\left\{\begin{array}{l}
D_{0}^{\mu} u(x, t)=1+v \frac{\mathrm{d} u}{\mathrm{~d} x}+u \\
D_{0}^{\mu} v(x, t)=1-u \frac{\mathrm{d} v}{\mathrm{~d} x}-v
\end{array}\right.
$$

subject to the initial conditions

$$
\begin{aligned}
& u(0, x)=e^{-x}, \\
& v(0, x)=e^{x} .
\end{aligned}
$$

is

The closed-form result of this arrangement when $\mu=1$

$$
\begin{aligned}
& u(t, x)=e^{t-x}, \\
& v(t, x)=e^{x-t} .
\end{aligned}
$$

Solution:

$$
\begin{aligned}
\text { Let } u(t, x)= & \sum_{k=0}^{\infty} a_{k}(x) t^{\mu k}=a_{0}(x)+t^{\mu} a_{1}(x)+t^{2 \mu} a_{2}(x) \\
& +\cdots+t^{n \mu} a_{n}(x)+\cdots, \\
v(t, x)= & \sum_{k=0}^{\infty} b_{k}(x) t^{\mu k}=b_{0}(x)+t^{\mu} b_{1}(x)+t^{2 \mu} b_{2}(x) \\
& +\cdots+t^{n \mu} b_{n}(x)+\cdots .
\end{aligned}
$$

So using Theorem 1, equations (12) and (13) become

$$
\begin{aligned}
D^{\mu}[u(x, t)] & =D^{\mu}\left(\sum_{k=0}^{\infty} a_{k}(x) t^{\mu k}\right), \\
\text { Or } D^{\mu}[u(x, t)] & =\sum_{k=1}^{\infty} a_{k}(x) \frac{\Gamma(k \mu+1)}{\Gamma((k-1) \mu+1)} t^{\mu(k-1)}, \\
D^{\mu}[v(x, t)] & =D^{\mu}\left(\sum_{k=0}^{\infty} b_{k}(x) t^{\mu k}\right), \\
\text { Or } D^{\mu}[v(x, t)] & =\sum_{k=1}^{\infty} \frac{\Gamma(k \mu+1)}{\Gamma((k-1) \mu+1)} b_{k}(x) t^{\mu k(k-1)} .
\end{aligned}
$$

Similarly,

$$
\begin{gathered}
S \frac{\partial u(x, t)}{\partial x}=\sum_{k=0}^{\infty} \frac{\partial a_{k}(x)}{\partial x} t^{\mu k}, \\
\frac{\partial v(x, t)}{\partial x}=\sum_{k=0}^{\infty} \frac{\partial b_{k}(x)}{\partial x} t^{\mu k} .
\end{gathered}
$$

Apply (12)-(17) on the first and second equation of system (9), respectively, system (9) becomes 


$$
\left\{\begin{array}{l}
\sum_{k=1}^{\infty} a_{k} \frac{\Gamma(k \mu+1)}{\Gamma((k-1) \mu+1)} t^{\mu(k-1)}=1+\sum_{k=0}^{\infty} t^{\mu k} a_{k}+\left(\sum_{k=0}^{\infty} t^{\mu k} \frac{\partial a_{k}}{\partial x}\right)\left(\sum_{k=0}^{\infty} b_{k} t^{\mu k}\right), \\
\sum_{k=1}^{\infty} \frac{\Gamma(k \mu+1)}{\Gamma((k-1) \mu+1)} b_{k} t^{\mu(k-1)}=1+\sum_{k=0}^{\infty} t^{\mu k} b_{k}+\left(\sum_{k=0}^{\infty} t^{\mu k} \frac{\partial b_{k}}{\partial x}\right)\left(\sum_{k=0}^{\infty} a_{k} t^{\mu k}\right) .
\end{array}\right.
$$

Comparing the different powers of $t$ to both sides of system (18), for $t^{0}$,

$$
\left\{\begin{array}{l}
a_{1}(x)=\frac{1}{\Gamma(\mu+1)}\left(1+b_{0} \frac{\partial a_{0}}{\partial x}+a_{0}\right) \\
b_{1}(x)=\frac{1}{\Gamma(\mu+1)}\left(1-a_{0} \frac{\partial b_{0}}{\partial x}-b_{0}\right)
\end{array}\right.
$$

But $a_{0}=u(0, x)=e^{-x}, v(0, x)=b_{0}=e^{x}, \partial a_{0} / \partial x=-e^{-x}$, and $\partial b_{0} / \partial x=-e^{x}$, so (19) becomes

$$
\left\{\begin{array}{l}
a_{1}(x)=\frac{1}{\Gamma(\mu+1)}\left(1+e^{x}\left(-e^{-x}\right)+e^{-x}\right)=\frac{e^{-x}}{\Gamma(\mu+1)} \\
b_{1}(x)=\frac{1}{\Gamma(\mu+1)}\left(1-e^{-x}\left(e^{x}\right)-e^{x}\right)=\frac{-e^{x}}{\Gamma(\mu+1)}
\end{array}\right.
$$

For $t^{\mu}$

$$
\left\{\begin{array}{l}
a_{2}(x)=\frac{\Gamma(\mu+1)}{\Gamma(2 \mu+1)}\left(a_{1}+b_{1} \frac{\partial a_{0}}{\partial x}+b_{0} \frac{\partial a_{1}}{\partial x}\right) \\
b_{2}(x)=\frac{\Gamma(\mu+1)}{\Gamma(2 \mu+1)}\left(-b_{1}-a_{1} \frac{\partial b_{0}}{\partial x}-a_{0} \frac{\partial b_{1}}{\partial x}\right) .
\end{array}\right.
$$

But $\partial a_{0} / \partial x=-e^{-x}, \partial b_{0} / \partial x=e^{x}, \partial a_{1} / \partial x=e^{-x} / \Gamma(\mu+1)$, $\partial b_{1} / \partial x=-e^{x} / \Gamma(\mu+1)$, so (21) becomes

$$
\left\{\begin{array}{l}
a_{2}(x)=\frac{\Gamma(\mu+1)}{\Gamma(2 \mu+1)}\left(\frac{e^{-x}}{\Gamma(\mu+1)}+e^{x}\left(\frac{-e^{-x}}{\Gamma(\mu+1)}\right)-\frac{e^{x}}{\Gamma(\mu+1)}\left(-e^{-x}\right)\right)=\frac{e^{-x}}{\Gamma(2 \mu+1)} \\
b_{2}(x)=\frac{\Gamma(\mu+1)}{\Gamma(2 \mu+1)}\left(\frac{e^{x}}{\Gamma(\mu+1)}-e^{-x}\left(\frac{-e^{x}}{\Gamma(\mu+1)}\right)-\frac{e^{-x}}{\Gamma(\mu+1)}\left(e^{x}\right)\right)=\frac{e^{x}}{\Gamma(2 \mu+1)}
\end{array}\right.
$$

For $t^{2 \mu}$,

$$
\left\{\begin{array}{l}
a_{3}(x)=\frac{\Gamma(2 \mu+1)}{\Gamma(3 \mu+1)}\left(a_{2}+b_{2} \frac{\partial a_{0}}{\partial x}+b_{0} \frac{\partial a_{2}}{\partial x}+b_{1} \frac{\partial a_{1}}{\partial x}\right) \\
b_{3}(x)=\frac{\Gamma(2 \mu+1)}{\Gamma(3 \mu+1)}\left(-b_{2}-a_{2} \frac{\partial b_{0}}{\partial x}-a_{0} \frac{\partial b_{2}}{\partial x}-a_{1} \frac{\partial b_{1}}{\partial x}\right) .
\end{array}\right.
$$

But $\partial a_{0} / \partial x=-e^{-x}, \partial b_{0} / \partial x=e^{x}, \partial a_{1} / \partial x=e^{-x} / \Gamma(\mu+1)$, $\partial b_{1} / \partial x=-e^{x} / \Gamma(\mu+1), \partial a_{2} / \partial x=-e^{-x} / \Gamma(2 \mu+1), \quad \partial b_{2} / \partial x=$ $e^{x} / \Gamma(2 \mu+1)$, so $(26)$ becomes

$$
\left\{\begin{array}{l}
a_{3}(x)=\frac{\Gamma(2 \mu+1)}{\Gamma(3 \mu+1)}\left(\frac{e^{-x}}{\Gamma(2 \mu+1)}+\left(\frac{-e^{x}}{\Gamma(\mu+1)}\right)\left(\frac{-e^{-x}}{\Gamma(\mu+1)}\right)+\frac{e^{x}}{\Gamma(2 \mu+1)}\left(-e^{-x}\right)+e^{x}\left(\frac{-e^{-x}}{\Gamma(2 \mu+1)}\right)\right), \\
b_{3}(x)=\frac{\Gamma(2 \mu+1)}{\Gamma(3 \mu+1)}\left(-\frac{e^{x}}{\Gamma(2 \mu+1)}-\frac{e^{-x}}{\Gamma(2 \mu+1)}\left(e^{x}\right)-e^{-x}\left(\frac{e^{x}}{\Gamma(2 \mu+1)}\right)-\frac{e^{-x}}{\Gamma(\mu+1)}\left(\frac{-e^{x}}{\Gamma(\mu+1)}\right)\right),
\end{array}\right.
$$


International Journal of Differential Equations

5

$$
\left\{\begin{array}{l}
a_{3}(x)=\frac{1}{\Gamma(3 \mu+1)}\left(e^{-x}-2+\frac{\Gamma(2 \mu+1)}{\Gamma(\mu+1)^{2}}\right), \\
b_{3}(x)=\frac{1}{\Gamma(3 \mu+1)}\left(-e^{x}-2+\frac{\Gamma(2 \mu+1)}{\Gamma(\mu+1)^{2}}\right) .
\end{array}\right.
$$

For $t^{3 \mu}$,

$$
\begin{aligned}
& \left\{\begin{array}{l}
a_{4}(x)=\frac{\Gamma(3 \mu+1)}{\Gamma(4 \mu+1)}\left(a_{3}+\frac{\partial a_{3}}{\partial x} b_{0}+\frac{\partial a_{2}}{\partial x} b_{1}+\frac{\partial a_{1}}{\partial x} b_{2}+\frac{\partial a_{0}}{\partial x} b_{3}\right), \\
b_{4}(x)=\frac{\Gamma(3 \mu+1)}{\Gamma(4 \mu+1)}\left(-b_{3}-\frac{\partial b_{3}}{\partial x} a_{0}-\frac{\partial b_{2}}{\partial x} a_{1}-\frac{\partial b_{1}}{\partial x} a_{2}-\frac{\partial b_{0}}{\partial x} a_{3}\right),
\end{array}\right. \\
& \left\{\begin{array}{l}
a_{4}(x)=\frac{-0.5}{\Gamma(4 \mu+1) \Gamma(2 \mu+(1 / 2)) \Gamma(\mu+1)^{2} \pi}\left(\left(\begin{array}{c}
-6 \pi e^{-x} \Gamma(\mu) \mu \Gamma\left(2 \mu+\frac{1}{2}\right)+4 \Gamma\left(2 \mu+\frac{1}{2}\right) \Gamma(\mu) \mu \pi+ \\
e^{-x} \Gamma\left(\mu+\frac{1}{4}\right) \Gamma\left(\mu+\frac{3}{4}\right) \Gamma\left(\mu+\frac{1}{2}\right) \sqrt{2} 16^{\mu}- \\
\Gamma\left(\mu+\frac{1}{4}\right) \Gamma\left(\mu+\frac{3}{4}\right) \Gamma\left(\mu+\frac{1}{2}\right) \sqrt{2} 16^{\mu}
\end{array}\right) \Gamma(\mu) \mu\right) \\
b_{4}(x)=\frac{-0.5}{\Gamma(4 \mu+1) \Gamma(2 \mu+(1 / 2)) \Gamma(\mu+1)^{2} \pi}\left(\begin{array}{c}
-6 \pi e^{-x} \Gamma(\mu) \mu \Gamma\left(2 \mu+\frac{1}{2}\right)+4 \Gamma\left(2 \mu+\frac{1}{2}\right) \Gamma(\mu) \mu \pi+ \\
e^{-x} \Gamma\left(\mu+\frac{1}{4}\right) \Gamma\left(\mu+\frac{3}{4}\right) \Gamma\left(\mu+\frac{1}{2}\right) \sqrt{2} 16^{\mu}+ \\
\Gamma\left(\mu+\frac{1}{4}\right) \Gamma\left(\mu+\frac{3}{4}\right) \Gamma\left(\mu+\frac{1}{2}\right) \sqrt{2} 16^{\mu}
\end{array}\right) \Gamma(\mu) \mu
\end{array}\right) .
\end{aligned}
$$




$$
\begin{aligned}
& u(t, x)=e^{-x}+\frac{e^{-x}}{\Gamma(\mu+1)} t^{\mu}+\frac{e^{-x}}{\Gamma(2 \mu+1)} t^{2 \mu}+\frac{1}{\Gamma(3 \mu+1)}\left(e^{-x}-2+\frac{\Gamma(2 \mu+1)}{(\Gamma(\mu+1))^{2}}\right) t^{3 \mu} \\
& +\left[\frac{-0.5}{\Gamma(4 \mu+1) \Gamma(2 \mu+(1 / 2)) \Gamma(\mu+1)^{2} \pi}\left(\left(\begin{array}{c}
-6 \pi e^{-x} \Gamma(\mu) \mu \Gamma\left(2 \mu+\frac{1}{2}\right)+4 \Gamma\left(2 \mu+\frac{1}{2}\right) \Gamma(\mu) \mu \pi+ \\
e^{-x} \Gamma\left(\mu+\frac{1}{4}\right) \Gamma\left(\mu+\frac{3}{4}\right) \Gamma\left(\mu+\frac{1}{2}\right) \sqrt{2} 16^{\mu}- \\
\Gamma\left(\mu+\frac{1}{4}\right) \Gamma\left(\mu+\frac{3}{4}\right) \Gamma\left(\mu+\frac{1}{2}\right) \sqrt{2} 16^{\mu}
\end{array}\right) \Gamma(\mu) \mu\right) t^{4 \mu}+\cdots,\right. \\
& v(t, x)=e^{-x}+\frac{e^{-x}}{\Gamma(\mu+1)} t^{\mu}+\frac{e^{-x}}{\Gamma(2 \mu+1)} t^{2 \mu}+\frac{1}{\Gamma(3 \mu+1)}\left(e^{-x}-2+\frac{\Gamma(2 \mu+1)}{(\Gamma(\mu+1))^{2}}\right) t^{3 \mu} \\
& +\left[\frac{-0.5}{\Gamma(4 \mu+1) \Gamma(2 \mu+(1 / 2)) \Gamma(\mu+1)^{2} \pi}\left(\left(\begin{array}{c}
-6 \pi e^{-x} \Gamma(\mu) \mu \Gamma\left(2 \mu+\frac{1}{2}\right)+4 \Gamma\left(2 \mu+\frac{1}{2}\right) \Gamma(\mu) \mu \pi+ \\
e^{-x} \Gamma\left(\mu+\frac{1}{4}\right) \Gamma\left(\mu+\frac{3}{4}\right) \Gamma\left(\mu+\frac{1}{2}\right) \sqrt{2} 16^{\mu}+ \\
\Gamma\left(\mu+\frac{1}{4}\right) \Gamma\left(\mu+\frac{3}{4}\right) \Gamma\left(\mu+\frac{1}{2}\right) \sqrt{2} 16^{\mu}
\end{array}\right) \Gamma(\mu) \mu\right) t^{4 \mu}+\cdots,\right.
\end{aligned}
$$

If $\mu=1$, then equations (27) and (28) give

$$
\begin{aligned}
& u(t, x)=e^{-x}\left(1+\frac{t}{\Gamma(2)}+\frac{t^{2}}{\Gamma(3)}+\frac{t^{3}}{\Gamma(4)}+\frac{t^{4}}{\Gamma(5)}+\ldots\right)=e^{t-x} \\
& v(t, x)=e^{x}\left(1-\frac{t}{\Gamma(2)}+\frac{t^{2}}{\Gamma(3)}-\frac{t^{3}}{\Gamma(4)}+\frac{t^{4}}{\Gamma(5)}+\ldots\right)=e^{-t+x}
\end{aligned}
$$

Example 2. Consider the following fractional-order system:

$$
\left\{\begin{array}{l}
D_{0}^{\mu} u(t, x)=2-2 v \frac{\mathrm{d} u}{\mathrm{~d} x}+u \\
D_{0}^{\mu} v(t, x)=3+3 u \frac{\mathrm{d} v}{\mathrm{~d} x}-v,
\end{array}\right.
$$

subject to the initial conditions

$$
\begin{aligned}
& u(0, x)=e^{x}, \\
& v(0, x)=e^{-x}
\end{aligned}
$$

The closed-form result of this system when $\mu=1$ is

$$
\begin{aligned}
& u(t, x)=e^{x+t}, \\
& v(t, x)=e^{-x-t} .
\end{aligned}
$$

Solution:

Let

$$
u(t, x)=\sum_{k=0}^{\infty} a_{k}(x) t^{\mu k}=a_{0}+t^{\mu} a_{1}+t^{2 \mu} a_{2}+\cdots+t^{n \mu} a_{n}+\cdots,
$$

$$
v(t, x)=\sum_{k=0}^{\infty} b_{k}(x) t^{\mu k}=b_{0}+t^{\mu} b_{1}+t^{2 \mu} b_{2}+\cdots+t^{n \mu} b_{n}+\cdots
$$

Apply (12)-(17) on the first and second equations of the system (30), respectively. So, system (30) becomes

$$
\left\{\begin{array}{l}
\sum_{k=1}^{\infty} a_{k} \frac{\Gamma(k \mu+1)}{\Gamma((k-1) \mu+1)} t^{\mu(k-1)}=2-2\left(\sum_{k=0}^{\infty} b_{k} t^{\mu k}\right)\left(\sum_{k=0}^{\infty} \frac{\partial a_{k}}{\partial x} t^{\mu k}\right)+\sum_{k=0}^{\infty} a_{k} t^{\mu k} \\
\sum_{k=1}^{\infty} b_{k} \frac{\Gamma(k \mu+1)}{\Gamma((k-1) \mu+1)} t^{\mu(k-1)}=3+3\left(\sum_{k=0}^{\infty} a_{k} t^{\mu k}\right)\left(\sum_{k=0}^{\infty} \frac{\partial b_{k}}{\partial x} t^{\mu k}\right)+\sum_{k=0}^{\infty} b_{k} t^{\mu k}
\end{array}\right.
$$


Comparing the different powers of $t$ to both sides of the system (35), for $t^{0}$,

$$
\left\{\begin{array}{l}
a_{1}(x)=\frac{1}{\Gamma(\mu+1)}\left(2+a_{0}-2 b_{0} \frac{\partial a_{0}}{\partial x}\right) \\
b_{1}(x)=\frac{1}{\Gamma(\mu+1)}\left(3-b_{0}+3 a_{0} \frac{\partial b_{0}}{\partial x}\right) .
\end{array}\right.
$$

After substituting the values $a_{0} b_{0}, \partial a_{0} / \partial x$, and $\partial b_{0} / \partial x$, we obtain

$$
\left\{\begin{array}{l}
a_{1}(x)=\frac{e^{x}}{\Gamma(\mu+1)} \\
b_{1}(x)=\frac{-e^{-x}}{\Gamma(\mu+1)}
\end{array}\right.
$$

For $t^{\mu}$,

$$
\left\{\begin{array}{l}
a_{2}(x)=\frac{\Gamma(\mu+1)}{\Gamma(2 \mu+1)}\left(a_{1}-2 b_{0} \frac{\partial a_{1}}{\partial x}-2 b_{1} \frac{\partial a_{0}}{\partial x}\right), \\
b_{2}(x)=\frac{\Gamma(\mu+1)}{\Gamma(2 \mu+1)}\left(-b_{1}+3 a_{1} \frac{\partial b_{0}}{\partial x}+3 a_{0} \frac{\partial b_{1}}{\partial x}\right) .
\end{array}\right.
$$

After substituting the values $a_{0} a_{1} b_{0}, b_{1}, \partial a_{0} / \partial x, \partial a_{1} / \partial x$, $\partial b_{0} / \partial x$, and $\partial b_{1} / \partial x$, we obtain

$$
\left\{\begin{array}{l}
a_{2}(x)=\frac{e^{x}}{\Gamma(2 \mu+1)} \\
b_{2}(x)=\frac{e^{-x}}{\Gamma(2 \mu+1)}
\end{array}\right.
$$

For $t^{2 \mu}$,

$$
\left\{\begin{array}{l}
a_{3}(x)=\frac{\Gamma(2 \mu+1)}{\Gamma(3 \mu+1)}\left(a_{2}-2 \frac{\partial a_{2}}{\partial x} b_{0}-2 \frac{\partial a_{1}}{\partial x} b_{1}-2 \frac{\partial a_{0}}{\partial x} b_{2}\right) \\
b_{3}(x)=\frac{\Gamma(2 \mu+1)}{\Gamma(3 \mu+1)}\left(-b_{2}+3 \frac{\partial b_{2}}{\partial x} a_{0}+3 \frac{\partial b_{1}}{\partial x} a_{1}+3 \frac{\partial b_{0}}{\partial x} a_{2}\right) .
\end{array}\right.
$$

After substituting the values $a_{0} a_{1}, a_{2} b_{0}, b_{1} b_{2}, \partial a_{0} / \partial x$, $\partial a_{1} / \partial x, \partial a_{2} / \partial x, \partial b_{0} / \partial x \partial b_{1} / \partial x$, and $\partial b_{2} / \partial x$, we obtain

$$
\left\{\begin{array}{l}
a_{3}(x)=\frac{1}{\Gamma(3 \mu+1)}\left(e^{x}-4+2 \frac{\Gamma(2 \mu+1)}{\Gamma(\mu+1)^{2}}\right), \\
b_{3}(x)=\frac{1}{\Gamma(3 \mu+1)}\left(-e^{-x}-6+3 \frac{\Gamma(2 \mu+1)}{\Gamma(\mu+1)^{2}}\right) .
\end{array}\right.
$$

For $t^{3 \mu}$

$$
\left\{\begin{array}{l}
a_{4}(x)=\frac{\Gamma(3 \mu+1)}{\Gamma(4 \mu+1)}\left(a_{3}-2 \frac{\partial a_{3}}{\partial x} b_{0}-2 \frac{\partial a_{2}}{\partial x} b_{1}-2 \frac{\partial a_{1}}{\partial x} b_{2}-2 \frac{\partial a_{0}}{\partial x} b_{3}\right) \\
b_{4}(x)=\frac{\Gamma(3 \mu+1)}{\Gamma(4 \mu+1)}\left(-b_{3}+3 \frac{\partial b_{3}}{\partial x} a_{0}+3 \frac{\partial b_{2}}{\partial x} a_{1}+3 \frac{\partial b_{1}}{\partial x} a_{2}+3 \frac{\partial b_{0}}{\partial x} a_{3}\right)
\end{array}\right.
$$

After inserting the values $a_{0} a_{1}, a_{2} a_{3} b_{0}, b_{1} b_{2} b_{3}, \partial a_{0} / \partial x$, $\partial a_{1} / \partial x, \partial a_{2} / \partial x \partial a_{3} / \partial x, \partial b_{0} / \partial x \partial b_{1} / \partial x, \partial b_{2} / \partial x$, and $\partial b_{3} / \partial x$, we obtain 
8

International Journal of Differential Equations

$$
\left\{\begin{array}{c}
a_{4}(x)=\frac{-1}{\Gamma(4 \mu+1) \Gamma(2 \mu+(1 / 2)) \Gamma(\mu+1)^{2} \pi}\left(\left(\begin{array}{c}
-13 \pi e^{x} \Gamma(\mu) \mu \Gamma\left(2 \mu+\frac{1}{2}\right)+4 \Gamma\left(2 \mu+\frac{1}{2}\right) \Gamma(\mu) \mu \pi+ \\
3 e^{x} \Gamma\left(\mu+\frac{1}{4}\right) \Gamma\left(\mu+\frac{3}{4}\right) \Gamma\left(\mu+\frac{1}{2}\right) \sqrt{2} 16^{\mu}- \\
\Gamma\left(\mu+\frac{1}{4}\right) \Gamma\left(\mu+\frac{3}{4}\right) \Gamma\left(\mu+\frac{1}{2}\right) \sqrt{2} 16^{\mu}
\end{array}\right) \Gamma(\mu) \mu\right) \\
b_{4}(x)=\frac{-0.5}{\Gamma(4 \mu+1) \Gamma(2 \mu+1 / 2) \Gamma(\mu+1)^{2} \pi}\left(\left(\begin{array}{c}
-26 \pi e^{-x} \Gamma(\mu) \mu \Gamma\left(2 \mu+\frac{1}{2}\right)-12 \Gamma\left(2 \mu+\frac{1}{2}\right) \Gamma(\mu) \mu \pi+ \\
6 e^{-x} \Gamma\left(\mu+\frac{1}{4}\right) \Gamma\left(\mu+\frac{3}{4}\right) \Gamma\left(\mu+\frac{1}{2}\right) \sqrt{2} 16^{\mu}+ \\
3 \Gamma\left(\mu+\frac{1}{4}\right) \Gamma\left(\mu+\frac{3}{4}\right) \Gamma\left(\mu+\frac{1}{2}\right) \sqrt{2} 16^{\mu}
\end{array}\right) \Gamma(\mu) \mu\right.
\end{array}\right) .
$$

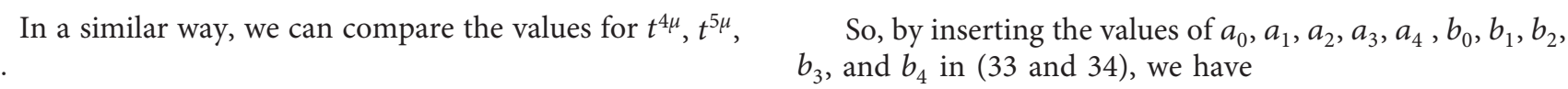

$$
\begin{aligned}
& u(t, x)=e^{x}+\frac{e^{x}}{\Gamma(\mu+1)} t^{\mu}+\frac{e^{x}}{\Gamma(2 \mu+1)} t^{2 \mu}+\frac{1}{\Gamma(3 \mu+1)}\left(e^{x}-4+2 \frac{\Gamma(2 \mu+1)}{\Gamma(\mu+1)^{2}}\right) t^{3 \mu} \\
& +\left[\frac{-1}{\Gamma(4 \mu+1) \Gamma(2 \mu+(1 / 2)) \Gamma(\mu+1)^{2} \pi}\left(\left(\begin{array}{c}
-13 \pi e^{x} \Gamma(\mu) \mu \Gamma\left(2 \mu+\frac{1}{2}\right)+4 \Gamma\left(2 \mu+\frac{1}{2}\right) \Gamma(\mu) \mu \pi+ \\
3 e^{x} \Gamma\left(\mu+\frac{1}{4}\right) \Gamma\left(\mu+\frac{3}{4}\right) \Gamma\left(\mu+\frac{1}{2}\right) \sqrt{2} 16^{\mu}- \\
\Gamma\left(\mu+\frac{1}{4}\right) \Gamma\left(\mu+\frac{3}{4}\right) \Gamma\left(\mu+\frac{1}{2}\right) \sqrt{2} 16^{\mu}
\end{array}\right) \Gamma(\mu) \mu\right) t^{4 \mu}+\cdots,\right.
\end{aligned}
$$

$$
\begin{aligned}
v(t, x)= & e^{-x}-\frac{e^{-x}}{\Gamma(\mu+1)} t^{\mu}+\frac{e^{-x}}{\Gamma(2 \mu+1)} t^{2 \mu}+\frac{1}{\Gamma(3 \mu+1)}\left(-e^{-x}-6+3 \frac{\Gamma(2 \mu+1)}{\Gamma(\mu+1)^{2}}\right) t^{3 \mu} \\
& +\left[\begin{array}{c}
-0.5 \\
\frac{\Gamma(4 \mu+1) \Gamma(2 \mu+(1 / 2)) \Gamma(\mu+1)^{2} \pi}{(4}\left(\begin{array}{c}
-26 \pi e^{-x} \Gamma(\mu) \mu \Gamma\left(2 \mu+\frac{1}{2}\right)-12 \Gamma\left(2 \mu+\frac{1}{2}\right) \Gamma(\mu) \mu \pi+ \\
6 e^{-x} \Gamma\left(\mu+\frac{1}{4}\right) \Gamma\left(\mu+\frac{3}{4}\right) \Gamma\left(\mu+\frac{1}{2}\right) \sqrt{2} 16^{\mu}+ \\
3 \Gamma\left(\mu+\frac{1}{4}\right) \Gamma\left(\mu+\frac{3}{4}\right) \Gamma\left(\mu+\frac{1}{2}\right) \sqrt{2} 16^{\mu}
\end{array}\right) \Gamma(\mu) \mu
\end{array}\right) t^{4 \mu}+\cdots
\end{aligned}
$$

If $\mu=1$, then equations (44) and (45) give 


$$
\begin{aligned}
& u(t, x)=\left(1+\frac{t}{\Gamma(2)}+\frac{t^{2}}{\Gamma(3)}+\frac{t^{3}}{\Gamma(4)}+\frac{t^{4}}{\Gamma(5)}+\cdots\right) e^{x}=e^{x+t} \\
& v(t, x)=\left(1-\frac{t}{\Gamma(2)}+\frac{t^{2}}{\Gamma(3)}-\frac{t^{3}}{\Gamma(4)}+\frac{t^{4}}{\Gamma(5)}+\cdots\right) e^{-x}=e^{-x-t}
\end{aligned}
$$

Example 3. Consider the following fractional-order system:

$$
\left\{\begin{array}{l}
D_{0}^{\mu} u(t, x)=2+3 u-v \frac{\mathrm{d} u}{\mathrm{~d} x} \\
D_{0}^{\mu} v(t, x)=2-3 v+u \frac{\mathrm{d} v}{\mathrm{~d} x}
\end{array}\right.
$$

subject to the initial conditions

$$
\begin{aligned}
& u(0, x)=e^{2 x}, \\
& v(0, x)=e^{-2 x} .
\end{aligned}
$$

The exact solution of this system when $\mu=1$ is

$$
\begin{aligned}
& u(t, x)=e^{2 x+3 t}, \\
& v(t, x)=e^{-2 x-3 t} .
\end{aligned}
$$

Solution:

Let

$$
\begin{aligned}
u(x, t)= & \sum_{k=0}^{\infty} a_{k}(x) t^{\mu k}=a_{0}(x)+t^{\mu} a_{1}(x)+t^{2 \mu} a_{2}(x) \\
& +\cdots+t^{n \mu} a_{n}(x)+\cdots, \\
v(x, t)= & \sum_{k=0}^{\infty} b_{k}(x) t^{\mu k}=b_{0}(x)+t^{\mu} b_{1}(x)+t^{2 \mu} b_{2}(x) \\
& +\cdots+t^{n \mu} b_{n}(x)+\cdots .
\end{aligned}
$$

Apply (12)-(17) on the first and second equations of the system (47), respectively, system (47) becomes

$$
\left\{\begin{array}{l}
\sum_{k=1}^{\infty} a_{k} \frac{\Gamma(k \mu+1)}{\Gamma((k-1) \mu+1)} t^{\mu(k-1)}=2-\left(\sum_{k=0}^{\infty} b_{k} t^{\mu k}\right)\left(\sum_{k=0}^{\infty} \frac{\partial a_{k}}{\partial x} t^{\mu k}\right)+3 \sum_{k=0}^{\infty} a_{k} t^{\mu k} \\
\sum_{k=1}^{\infty} b_{k} \frac{\Gamma(k \mu+1)}{\Gamma((k-1) \mu+1)} t^{\mu(k-1)}=2+\left(\sum_{k=0}^{\infty} a_{k} t^{\mu k}\right)\left(\sum_{k=0}^{\infty} \frac{\partial b_{k}}{\partial x} t^{\mu k}\right)+3 \sum_{k=0}^{\infty} b_{k} t^{\mu k}
\end{array}\right.
$$

Comparing the different powers of $t$ to both sides of the system (52), for $t^{0}$,

$$
\left\{\begin{array}{l}
a_{1}(x)=\frac{1}{\Gamma(\mu+1)}\left(2-b_{0} \frac{\partial a_{0}}{\partial x}+3 a_{0}\right) \\
b_{1}(x)=\frac{1}{\Gamma(\mu+1)}\left(2+a_{0} \frac{\partial b_{0}}{\partial x}-3 b_{0}\right)
\end{array}\right.
$$

After substituting the values $a_{0}(x) b_{0}(x), \partial a_{0}(x) / \partial x$, and $\partial b_{0}(x) / \partial x$, we obtain

$$
\left\{\begin{array}{l}
a_{1}(x)=\frac{3 e^{2 x}}{\Gamma(\mu+1)} \\
b_{1}(x)=\frac{-3 e^{-2 x}}{\Gamma(\mu+1)}
\end{array}\right.
$$

For $t^{\mu}$,

$$
\left\{\begin{array}{l}
a_{2}(x)=\frac{\Gamma(\mu+1)}{\Gamma(2 \mu+1)}\left(3 a_{1}-\frac{\partial a_{0}}{\partial x} b_{1}-\frac{\partial a_{1}}{\partial x} b_{0}\right), \\
b_{2}(x)=\frac{\Gamma(\mu+1)}{\Gamma(2 \mu+1)}\left(-3 b_{1}+\frac{\partial b_{0}}{\partial x} a_{1}+\frac{\partial b_{1}}{\partial x} a_{0}\right) .
\end{array}\right.
$$

After substituting the values $a_{0} a_{1} b_{0}, b_{1}, \partial a_{0} / \partial x, \partial a_{1} / \partial x$, $\partial b_{0} / \partial x$, and $\partial b_{1} / \partial x$, we obtain

$$
\left\{\begin{array}{l}
a_{2}(x)=\frac{9 e^{2 x}}{\Gamma(2 \mu+1)} \\
b_{2}(x)=\frac{9 e^{-2 x}}{\Gamma(2 \mu+1)}
\end{array}\right.
$$

For $t^{2 \mu}$ 


$$
\left\{\begin{array} { l } 
{ a _ { 3 } ( x ) = \frac { \Gamma ( 2 \mu + 1 ) } { \Gamma ( 3 \mu + 1 ) } ( 3 a _ { 2 } - \frac { \partial a _ { 2 } } { \partial x } b _ { 0 } - \frac { \partial a _ { 1 } } { \partial x } b _ { 1 } - \frac { \partial a _ { 0 } } { \partial x } b _ { 2 } ) , } \\
{ b _ { 3 } ( x ) = \frac { \Gamma ( 2 \mu + 1 ) } { \Gamma ( 3 \mu + 1 ) } ( - 3 b _ { 2 } + \frac { \partial b _ { 2 } } { \partial x } a _ { 0 } + \frac { \partial b _ { 1 } } { \partial x } a _ { 1 } + \frac { \partial b _ { 0 } } { \partial x } a _ { 2 } ) . }
\end{array} \quad \left\{\begin{array}{l}
a_{3}(x)=\frac{9}{\Gamma(3 \mu+1)}\left(3 e^{2 x}-4+2 \frac{\Gamma(2 \mu+1)}{\Gamma(\mu+1)^{2}}\right) \\
b_{3}(x)=\frac{9}{\Gamma(3 \mu+1)}\left(-3 e^{-2 x}-4+2 \frac{\Gamma(2 \mu+1)}{\Gamma(\mu+1)^{2}}\right) .
\end{array}\right.\right.
$$

After substituting the values $a_{0} a_{1}, a_{2} b_{0}, b_{1} b_{2}, \partial a_{0} / \partial x$, For $t^{3 \mu}$ $\partial a_{1} / \partial x, \partial a_{2} / \partial x, \partial b_{0} / \partial x \partial b_{1} / \partial x$, and $\partial b_{2} / \partial x$, we obtain

$$
\left\{\begin{array}{l}
a_{4}(x)=\frac{\Gamma(3 \mu+1)}{\Gamma(4 \mu+1)}\left(3 a_{3}-\frac{\partial a_{3}}{\partial x} b_{0}-\frac{\partial a_{2}}{\partial x} b_{1}-\frac{\partial a_{1}}{\partial x} b_{2}-\frac{\partial a_{0}}{\partial x} b_{3}\right) \\
b_{4}(x)=\frac{\Gamma(3 \mu+1)}{\Gamma(4 \mu+1)}\left(-3 b_{3}+\frac{\partial b_{3}}{\partial x} a_{0}+\frac{\partial b_{2}}{\partial x} a_{1}+\frac{\partial b_{1}}{\partial x} a_{2}+\frac{\partial b_{0}}{\partial x} a_{3}\right)
\end{array}\right.
$$

After inserting the values $a_{0} a_{1}, a_{2} a_{3} b_{0}, b_{1} b_{2} b_{3}, \partial a_{0} / \partial x$, $\partial a_{1} / \partial x, \partial a_{2} / \partial x \partial a_{3} / \partial x, \partial b_{0} / \partial x \partial b_{1} / \partial x, \partial b_{2} / \partial x$, and $\partial b_{3} / \partial x$, we obtain

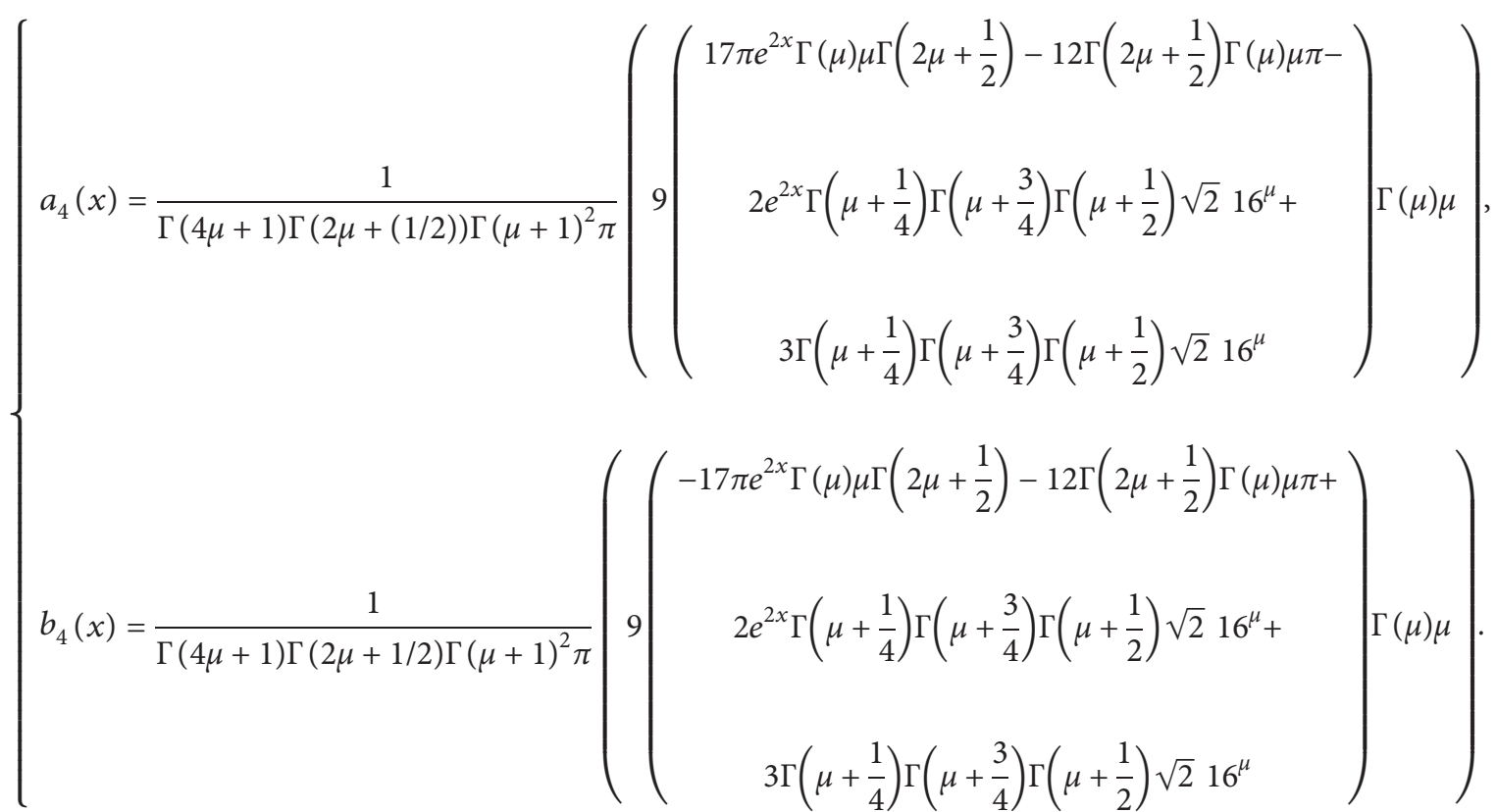

In a similar way, we can compare the values for $t^{4 \mu}, t^{5 \mu}$,

So, by inserting the values of $a_{0}, a_{1}, a_{2}, a_{3}, a_{4}, b_{0}, b_{1}, b_{2}$, $b_{3}$, and $b_{4}$ in (51) and (52), we have 
TABle 1: Numerical values for $u(t, x)$ of equation (27) for different values of $t$ and $x$.

\begin{tabular}{ccccccc}
\hline$x$ & $t$ & $\mu=1$ & $\mu=0.8$ & $\mu=0.6$ & $\mu=0.4$ & $\mu=0.2$ \\
\hline & 0.25 & 1.051264145 & 1.177800483 & 1.376647053 & 1.690057985 & 2.190896007 \\
0.2 & 0.50 & 1.349626476 & 1.552782962 & 1.825654773 & 2.178405687 & 2.598177788 \\
& 0.75 & 1.731407662 & 1.995745862 & 2.305360890 & 2.636753607 & 2.913559185 \\
& 1 & 2.217395789 & 2.524361162 & 2.833631770 & 3.091314344 & 3.183670371 \\
\hline & 0.25 & 0.778795634 & 0.871298353 & 1.010006624 & 1.199502045 & 1.416439046 \\
0.5 & 0.50 & 0.999827884 & 1.143252515 & 1.314743942 & 1.481305224 & 1.592001081 \\
& 0.75 & 1.282658343 & 1.458459258 & 1.623729060 & 1.723827348 & 1.717670616 \\
& 1 & 1.642687203 & 1.827732063 & 1.949469341 & 1.949842216 & 1.819993633 \\
\hline & 0.25 & 0.576945996 & 0.644235991 & 0.738392714 & 0.836089264 & 0.842707220 \\
0.8 & 0.50 & 0.740690714 & 0.839864902 & 0.936251891 & 0.964880499 & 0.846607044 \\
& 0.75 & 0.950216672 & 1.060427554 & 1.118763783 & 1.047514936 & 0.831734576 \\
& 1 & 1.216932611 & 1.311656534 & 1.294465704 & 1.104218862 & 0.809757060 \\
\hline
\end{tabular}

$$
\left.\left.\begin{array}{rl}
u(x, t)= & e^{2 x}+\frac{3 e^{2 x}}{\Gamma(\mu+1)} t^{\mu}+\frac{9 e^{2 x}}{\Gamma(2 \mu+1)} t^{2 \mu}+\frac{9}{\Gamma(3 \mu+1)}\left(3 e^{2 x}-4+2 \frac{\Gamma(2 \mu+1)}{\Gamma(\mu+1)^{2}}\right) t^{3 \mu} \\
& +\left[\frac{1}{\Gamma(4 \mu+1) \Gamma(2 \mu+(1 / 2)) \Gamma(\mu+1)^{2} \pi}\left(\left(\begin{array}{c}
17 \pi e^{2 x} \Gamma(\mu) \mu \Gamma\left(2 \mu+\frac{1}{2}\right)-12 \Gamma\left(2 \mu+\frac{1}{2}\right) \Gamma(\mu) \mu \pi- \\
2 e^{2 x} \Gamma\left(\mu+\frac{1}{4}\right) \Gamma\left(\mu+\frac{3}{4}\right) \Gamma\left(\mu+\frac{1}{2}\right) \sqrt{2} 16^{\mu}+ \\
3 \Gamma\left(\mu+\frac{1}{4}\right) \Gamma\left(\mu+\frac{3}{4}\right) \Gamma\left(\mu+\frac{1}{2}\right) \sqrt{2} 16^{\mu}
\end{array}\right)\right) \Gamma(\mu) \mu\right) t^{4 \mu}+\cdots
\end{array}\right)\right]
$$

$$
\begin{aligned}
& v(x, t)=e^{-2 x}-\frac{3 e^{-2 x}}{\Gamma(\mu+1)} t^{\mu}+\frac{9 e^{-2 x}}{\Gamma(2 \mu+1)} t^{2 \mu}+\frac{9}{\Gamma(3 \mu+1)}\left(-3 e^{-2 x}-4+2 \frac{\Gamma(2 \mu+1)}{\Gamma(\mu+1)^{2}}\right) t^{3 \mu} \\
& +\left[\frac{1}{\Gamma(4 \mu+1) \Gamma(2 \mu+(1 / 2)) \Gamma(\mu+1)^{2} \pi}\left(9\left(\begin{array}{c}
-17 \pi e^{2 x} \Gamma(\mu) \mu \Gamma\left(2 \mu+\frac{1}{2}\right)-12 \Gamma\left(2 \mu+\frac{1}{2}\right) \Gamma(\mu) \mu \pi+ \\
2 e^{2 x} \Gamma\left(\mu+\frac{1}{4}\right) \Gamma\left(\mu+\frac{3}{4}\right) \Gamma\left(\mu+\frac{1}{2}\right) \sqrt{2} 16^{\mu}+ \\
3 \Gamma\left(\mu+\frac{1}{4}\right) \Gamma\left(\mu+\frac{3}{4}\right) \Gamma\left(\mu+\frac{1}{2}\right) \sqrt{2} 16^{\mu}
\end{array}\right) \Gamma(\mu) \mu\right) t^{4 \mu}+\cdots .\right.
\end{aligned}
$$

If $\mu=1$, then equations (61) and (62) give

$$
\begin{aligned}
& u(x, t)=e^{2 x}\left(1+\frac{3 t}{\Gamma(2)}+\frac{9 t^{2}}{\Gamma(3)}+\frac{27 t^{3}}{\Gamma(4)}+\frac{81 t^{4}}{\Gamma(5)}+\cdots\right)=e^{2 x+3 t} \\
& v(x, t)=e^{-2 x}\left(1-\frac{3 t}{\Gamma(2)}+\frac{9 t^{2}}{\Gamma(3)}-\frac{27 t^{3}}{\Gamma(4)}+\frac{81 t^{4}}{\Gamma(5)}-\cdots\right)=e^{-2 x-3 t}
\end{aligned}
$$

\section{Results and Discussion}

Tables 1and 2 show the values of $u(t, x)$ and $v(t, x)$ for Example 1 with different values of $x, t$, and $\mu$. Afterward, the graphical simulations are drawn with the help of MATLAB. The graphs are shown in $2 \mathrm{D}$ and $3 \mathrm{D}$, and these can be seen in Figures 1 and 2. Tables 3 and 4 elaborate the values of $u(t, x)$ and $v(t, x)$ for Example 2 and graphical imitations can be 
TABle 2: Numerical values for $v(t, x)$ of equation (28) for different values of $t$ and $x$.

\begin{tabular}{ccccccc}
\hline$x$ & $t$ & $\mu=1$ & $\mu=0.8$ & $\mu=0.6$ & $\mu=0.4$ & $\mu=0.2$ \\
\hline & 0.25 & 0.951238964 & 0.865889787 & 0.777138934 & 0.743944913 & 1.125397015 \\
0.2 & 0.50 & 0.741111569 & 0.678152261 & 0.651799698 & 0.826638198 & 1.556286290 \\
& 0.75 & 0.579092812 & 0.557061297 & 0.638677023 & 1.066582027 & 1.987000806 \\
& 1 & 0.458026034 & 0.494869468 & 0.736296772 & 1.429900092 & 2.411626265 \\
\hline & 0.25 & 1.284038295 & 1.170123705 & 1.057085400 & 1.031611960 & 1.563206998 \\
0.5 & 0.50 & 1.000395979 & 0.921508202 & 0.903205690 & 1.162805382 & 2.141116646 \\
& 0.75 & 0.781693532 & 0.766339970 & 0.902730012 & 1.497237306 & 2.711609503 \\
& 1 & 0.618270477 & 0.693434087 & 1.050515555 & 1.989643341 & 3.270439580 \\
\hline & 0.25 & 1.733270400 & 1.580796538 & 1.434973603 & 1.419921858 & 2.154188653 \\
0.8 & 0.50 & 1.350393324 & 1.250004362 & 1.242568283 & 1.616583612 & 2.930555054 \\
& 0.75 & 1.055175900 & 1.048836629 & 1.259164265 & 2.078561123 & 3.689728934 \\
& 1 & 0.834577848 & 0.961468288 & 1.474666548 & 2.745217689 & 4.429716292 \\
\hline
\end{tabular}
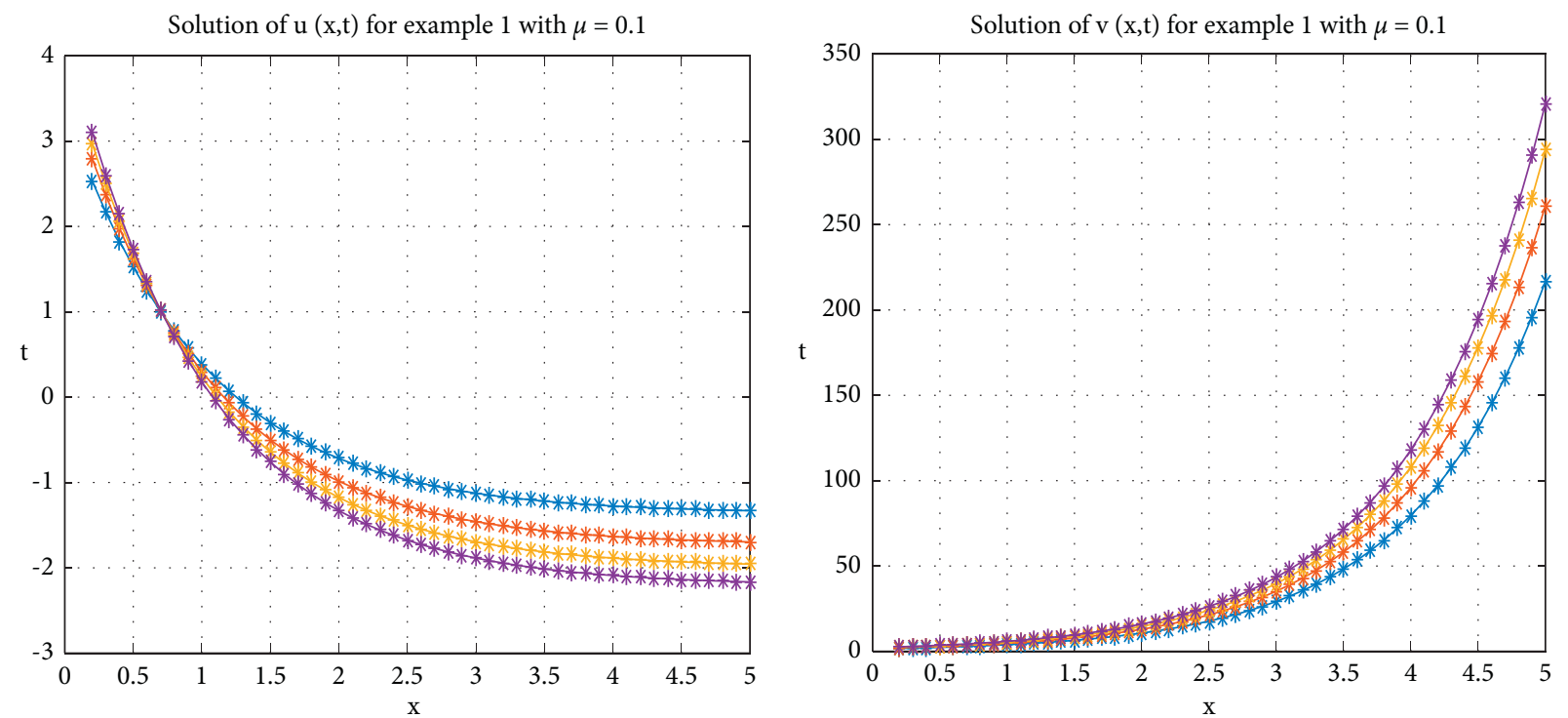

Figure 1: 2D graphical representation for $u(t, x)$ and $v(t, x)$ of equation (9).

Solution of $\mathrm{u}(\mathrm{x}, \mathrm{t})$ for example 1 with $\mu=0.1$

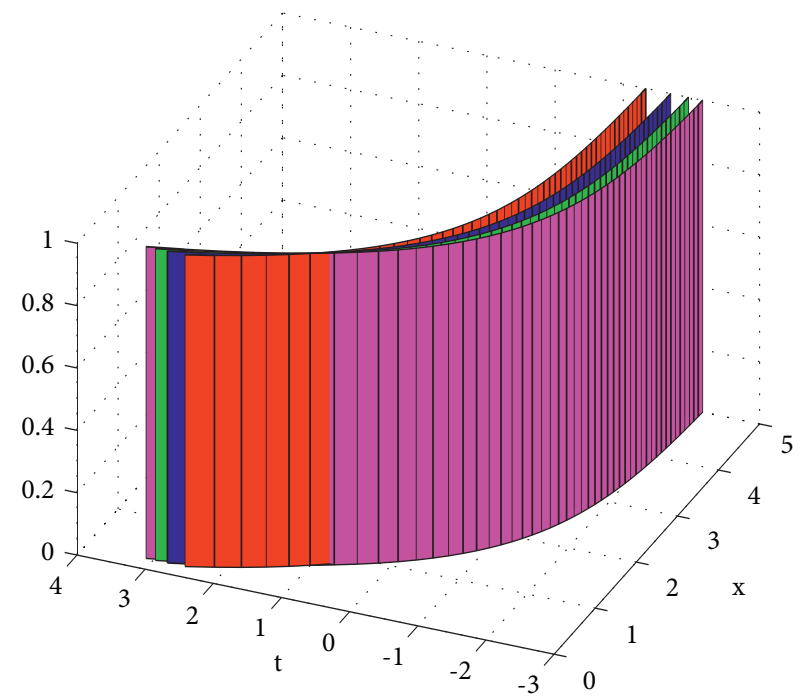

Solution of $\mathrm{v}(\mathrm{x}, \mathrm{t})$ for example 1 with $\mu=0.1$

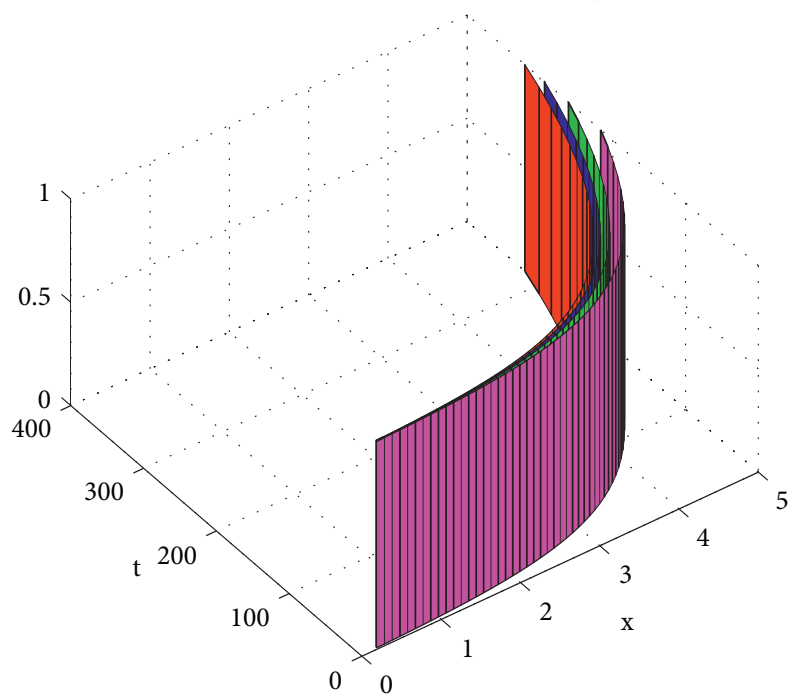

FIGURE 2: 3D graphical representation for $u(t, x)$ and $v(t, x)$ of equation (9). 
TABle 3: Numerical values for $u(t, x)$ of equation (44) for different values of $t$ and $x$.

\begin{tabular}{ccccccc}
\hline$x$ & $t$ & $\mu=1$ & $\mu=0.8$ & $\mu=0.6$ & $\mu=0.4$ & $\mu=0.2$ \\
\hline & 0.25 & 1.568301816 & 1.757926562 & 2.080012379 & 2.798019675 & 4.912818944 \\
0.2 & 0.50 & 2.013406109 & 2.332758728 & 2.890179752 & 4.141145675 & 6.791936646 \\
& 0.75 & 2.582956711 & 3.048410210 & 3.910944981 & 5.685815446 & 8.418001219 \\
& 1 & 3.307965802 & 3.959963255 & 5.203632802 & 7.434136691 & 9.906507037 \\
\hline & 0.25 & 2.116986021 & 2.376294107 & 2.834284463 & 3.918730674 & 7.189418490 \\
0.5 & 0.50 & 2.717813970 & 3.168001876 & 4.003206759 & 5.947667531 & 10.06655772 \\
& 0.75 & 3.486626866 & 4.168986944 & 5.506335361 & 8.294708222 & 12.55300584 \\
& 1 & 4.465286775 & 5.459753157 & 7.428416392 & 10.95364021 & 14.82625765 \\
\hline & 0.25 & 2.857632224 & 3.211002984 & 3.852445275 & 5.431532282 & 10.26250643 \\
0.8 & 0.50 & 3.668665124 & 4.295462192 & 5.505636061 & 8.386216959 & 14.48683382 \\
& 0.75 & 4.706453984 & 5.681607311 & 7.659887116 & 11.81634511 & 18.13467822 \\
& 1 & 6.027506680 & 7.484257760 & 10.43156010 & 15.70447300 & 21.46722633 \\
\hline
\end{tabular}

TABLE 4: Numerical values for $v(t, x)$ of equation (45) for different values of $t$ and $x$.

\begin{tabular}{ccccccc}
\hline$x$ & $t$ & $\mu=1$ & $\mu=0.8$ & $\mu=0.6$ & $\mu=0.4$ & $\mu=0.2$ \\
\hline & 0.25 & 0.637634547 & 0.574001290 & 0.497834882 & 0.570826497 & 1.834670972 \\
0.2 & 0.50 & 0.496781941 & 0.434184061 & 0.442592615 & 1.013033316 & 3.166481657 \\
& 0.75 & 0.388177520 & 0.351599173 & 0.584512413 & 1.808277898 & 4.444360353 \\
& 1 & 0.307024032 & 0.348139360 & 0.967799336 & 2.901554369 & 5.680755396 \\
\hline \multirow{4}{*}{0.5} & 0.25 & 0.472371290 & 0.422353092 & 0.350897578 & 0.362002598 & 1.261192050 \\
& 0.50 & 0.368025114 & 0.308098047 & 0.275946070 & 0.646105894 & 2.256111494 \\
& 0.75 & 0.287568980 & 0.228499125 & 0.342771994 & 1.211809268 & 3.227036363 \\
& 1 & 0.227448997 & 0.201390564 & 0.591130501 & 2.017332508 & 4.174882213 \\
\hline & 0.25 & 0.349941259 & 0.310009344 & 0.242043747 & 0.207302048 & 0.836348419 \\
& 0.50 & 0.272639710 & 0.214691230 & 0.152491273 & 0.374279373 & 1.581692701 \\
& 0.75 & 0.213036340 & 0.137304367 & 0.163686284 & 0.766993444 & 2.325220576 \\
& 1 & 0.168498362 & 0.092676381 & 0.312087366 & 1.362284840 & 3.059303926 \\
\hline
\end{tabular}
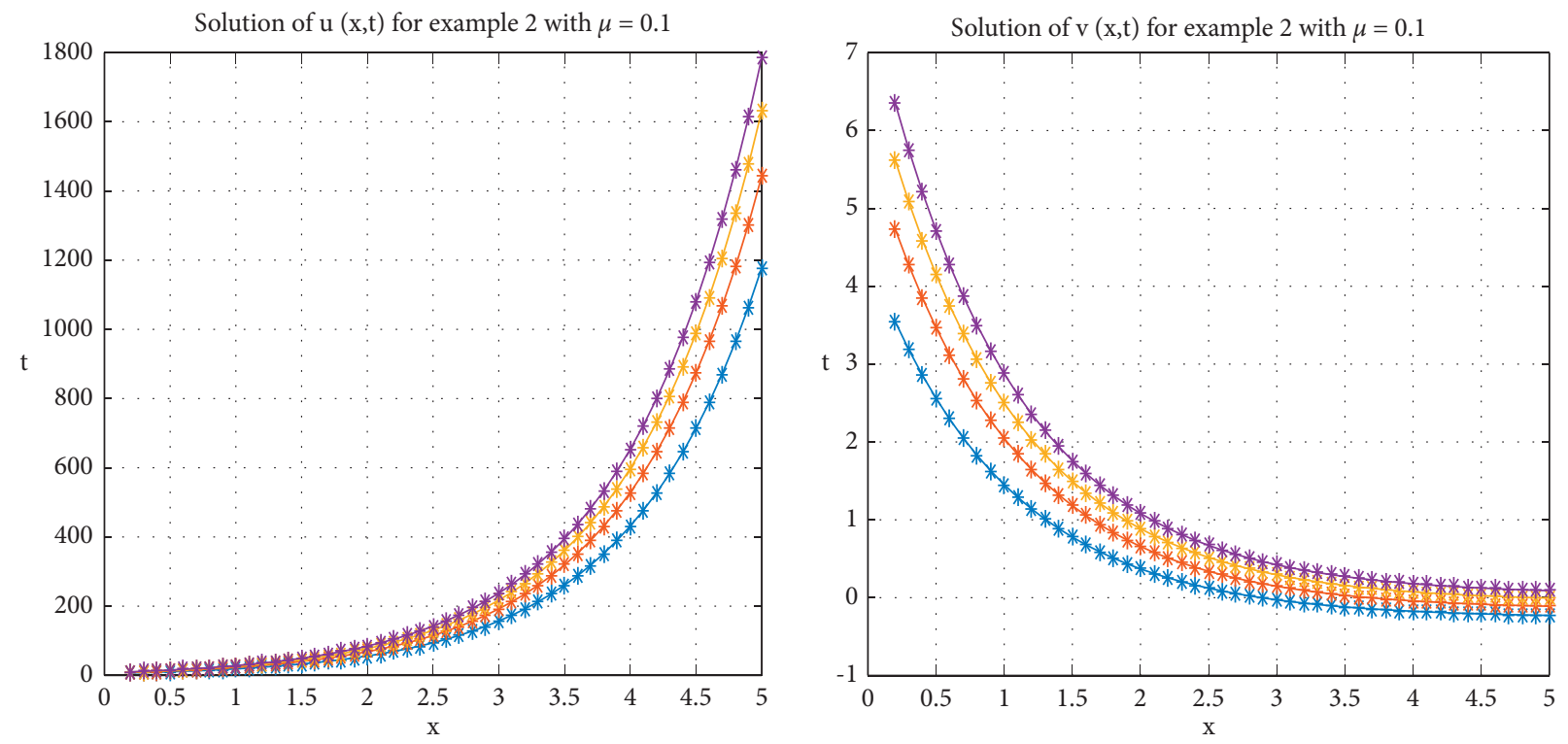

Figure 3: 2D graphical representation for $u(t, x)$ and $v(t, x)$ of equation (30).

seen in Figures 3 and 4 for different values of $t, x$, and $\mu$. Besides these, Tables 5 and 6 represent the values of $t, x$, and $\mu$ and simulations can be viewed in Figures 5 and 6 .
Fractional physical equations can be solved successfully by using analytical and approximate solutions method called fractional power series method (FPSM). The Caputo 

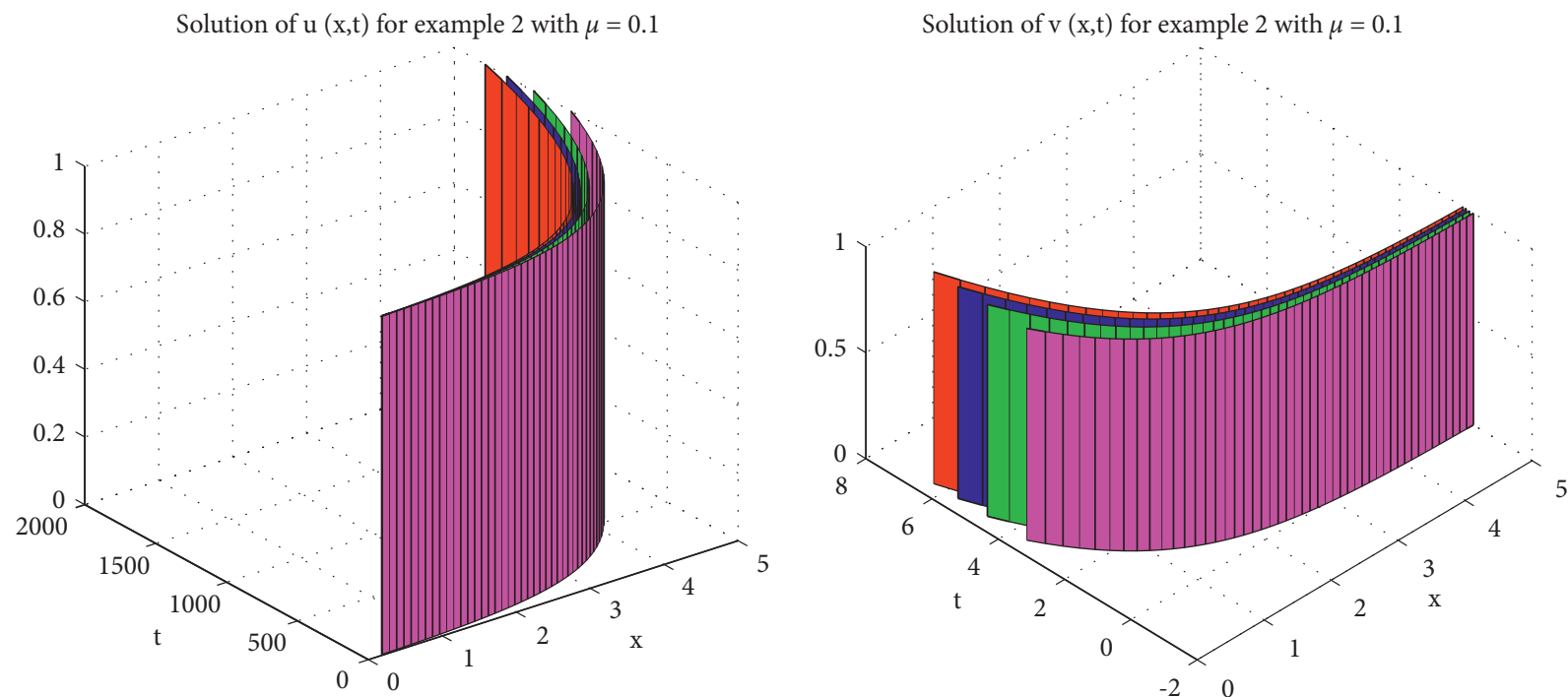

Figure 4: 3D graphical representation for $u(t, x)$ and $v(t, x)$ of equation (30).

TABle 5: Numerical values for $u(\mathrm{x}, \mathrm{t})$ of equation (61) for different values of $x$ and $t$.

\begin{tabular}{ccccccc}
\hline$x$ & $t$ & $\mu=1$ & $\mu=0.8$ & $\mu=0.6$ & $\mu=0.4$ & $\mu=0.2$ \\
\hline & 0.25 & 3.154830453 & 4.691959285 & 8.866135776 & 22.72166785 & 67.89003771 \\
0.2 & 0.50 & 6.561697696 & 11.20075599 & 22.75683011 & 51.52487616 & 108.6954101 \\
& 0.75 & 13.04982396 & 23.14398543 & 44.97510977 & 87.11237439 & 144.2819487 \\
& 1 & 24.42862944 & 42.63967701 & 76.52840205 & 128.5195969 & 176.9305414 \\
\hline & 0.25 & 5.748475877 & 8.635877638 & 16.93781919 & 46.24073749 & 145.4330455 \\
0.5 & 0.50 & 11.95619272 & 20.95933710 & 44.78616917 & 107.0281709 & 234.3493828 \\
& 0.75 & 23.77832957 & 43.84935668 & 89.83870690 & 182.5069498 & 311.9830971 \\
& 1 & 44.51186494 & 81.45614191 & 154.1476188 & 270.5167864 & 383.2455096 \\
\hline & 0.25 & 10.47440597 & 15.82216542 & 31.64538531 & 89.09527647 & 2867256176 \\
0.8 & 0.50 & 21.78560356 & 38.74063122 & 84.92624204 & 208.1617677 & 463.3058486 \\
& 0.75 & 43.32694134 & 81.57700295 & 171.5855108 & 356.3271994 & 617.5545123 \\
& 1 & 81.10590594 & 152.1843524 & 295.5790527 & 529.2525354 & 759.1758921 \\
\hline
\end{tabular}

Table 6: The numerical value for $v(\mathrm{x}, \mathrm{t})$ of equation (62) for different values of $x$ and $t$.

\begin{tabular}{ccccccc}
\hline$x$ & $t$ & $\mu=1$ & $\mu=0.8$ & $\mu=0.6$ & $\mu=0.4$ & $\mu=0.2$ \\
\hline & 0.25 & 0.317812874 & 0.247943726 & 0.525872181 & 4.855612927 & 31.24156799 \\
0.2 & 0.50 & 0.183290638 & 0.416943067 & 3.086090208 & 16.96966349 & 57.09462535 \\
& 0.75 & 0.302102248 & 1.709140335 & 9.532343390 & 34.85940052 & 81.01323439 \\
& 1 & 0.921690063 & 5.080281660 & 20.90355020 & 57.73320775 & 103.7181425 \\
\hline & 0.25 & 0.174419403 & 0.114746644 & 0.222808556 & 3.038942776 & 21.57352776 \\
0.5 & 0.50 & 0.100592035 & 0.167462940 & 1.791174215 & 11.28469761 & 39.88834029 \\
& 0.75 & 0.165797228 & 0.897605834 & 5982261590 & 23.65380070 & 56.90494333 \\
& 1 & 0.505834231 & 2.934839500 & 13.53544442 & 39.58514034 & 73.09600369 \\
\hline & 0.25 & 0.095723398 & 0.041646536 & 0.056483713 & 2.041933069 & 16.26759478 \\
0.8 & 0.50 & 0.055206079 & 0.030545346 & 1.080509256 & 8.164722200 & 30.44533086 \\
& 0.75 & 0.090991448 & 0.452226258 & 4.033935410 & 17.50403717 & 43.67403279 \\
& 1 & 0.277607711 & 1.757395880 & 9.491742250 & 29.62526973 & 56.29021759 \\
\hline
\end{tabular}

operator is presented as fractional derivatives. As compared to other methods to solve nonlinear equations, the power series method is employed efficiently to obtain the solution.
Results thus obtained show that FPSM can be applied to solve the system of nonlinear partial differential equations (PDEs) with accuracy and effectiveness. 

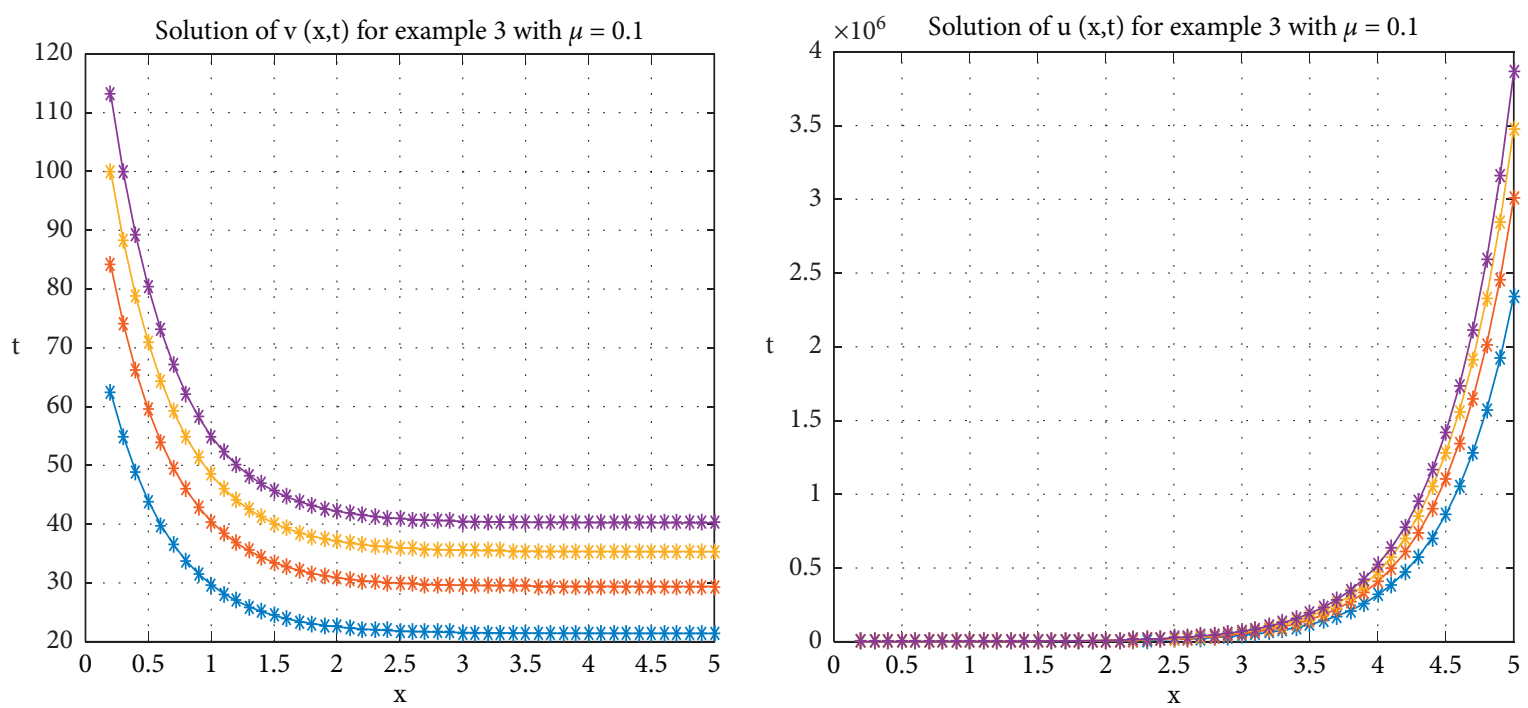

FIGURE 5: 2D graphical representation for $u(x, t)$ and $v(x, t)$ of equation (47).
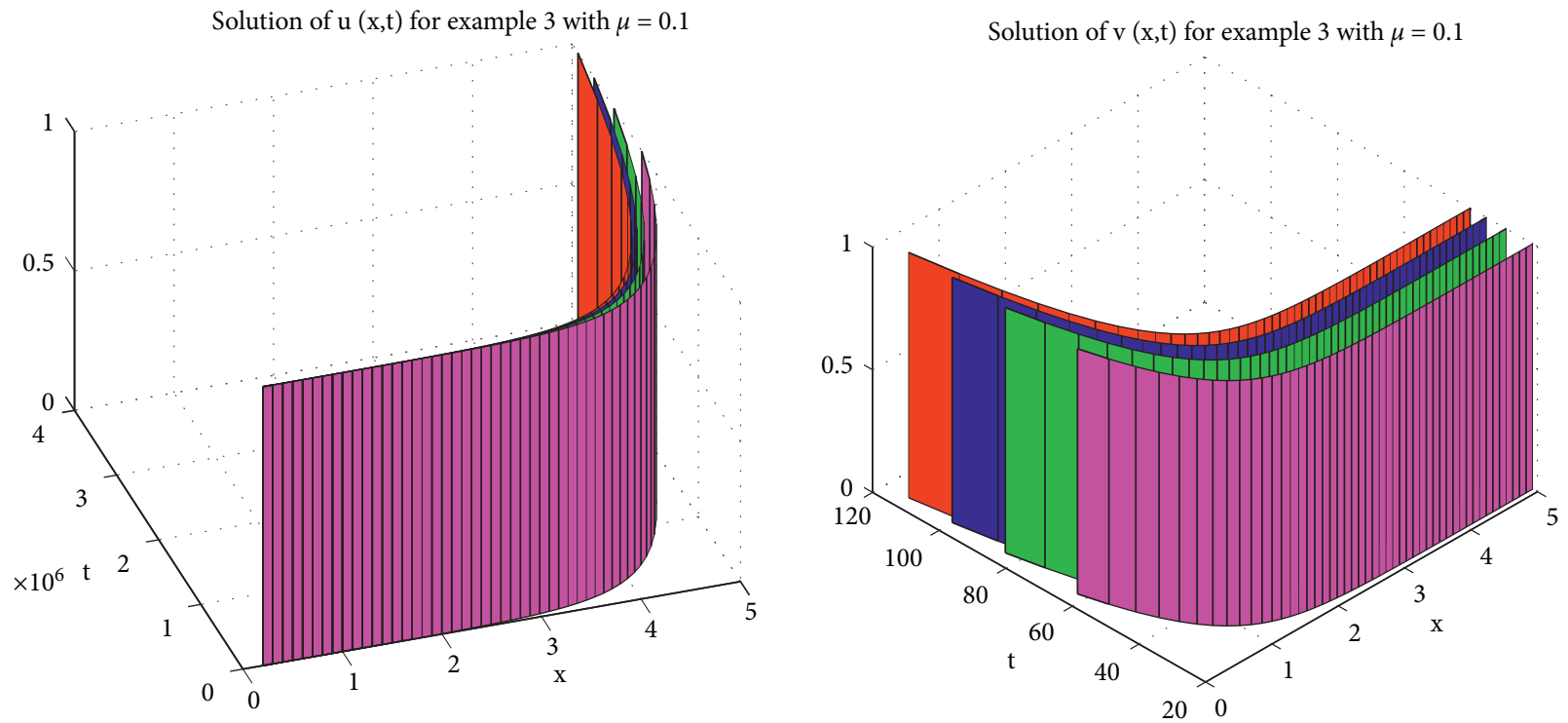

FIGURE 6: 3D graphical representation for $u(x, t)$ and $v(x, t)$ of equation (47).

\section{Conclusion}

Numerical simulation results prove the validity and reliability of the power series method. Therefore, it is more convenient to describe that PSM is very powerful in constructing fractional power series solutions for fractional PDEs in scientific problems of any order and time of space. As per the work done, we had offered the fractional-order power series method for finding the nonlinear fractionalorder partial differential equation with comparable easy implementation. In the tables, reasonable values are presented for fractional and integer cases. 2D and 3D graphical presentations are given for fractional-order $\mu=0.1$ for $\mathbf{t}=0.25,0.5,0.75$, and 1 . The proposed method is quite promising in reducing the computational cost of solving such problems to a great extent.

\section{Data Availability}

All the data are available in the article and cited wherever required.

\section{Conflicts of Interest}

The authors declare that they have no conflicts of interest. 


\section{References}

[1] D. G. Zill, The First Course in Differential Equations - the Classic, Brooks/Cole, Pacific Grove, CA, USA, 5th edition, 2001.

[2] L. Yilmaz, "Some Considerations on the series solution of Differential equations and its engineering applications," $R M Z$ Materials and GeoEnvironment, vol. 53, no. 1, pp. 247-259, 2006.

[3] A. El-Ajou, O. A. Arqub, and S. Mombackani, "Approximate analytical solution of the nonlinear fractional KdV-Burgers equation: a new iterative algorithm," Journal of Computational Physics, vol. 293, pp. 81-95, 2015.

[4] C. Lin, C.-W. Wang, and X.-L. Zhang, "Series solution for the Schrõdinger equation with a long-range spherically symmetric potential," Proceedings of the Royal Society of London. Series A: Mathematical, Physical and Engineering Sciences, vol. 458, no. 2022, pp. 1285-1290, 2002.

[5] L. Chang and M.-m. Lin, "Series solution of the differential equation determining the nth-shell one-electron density of a bare Coulomb problem in quantum physics," Communications in Nonlinear Science and Numerical Simulation, vol. 13, pp. 677-681, 2008.

[6] P. D. Lax, "Integrals of nonlinear equations of evolution and solitary waves," Communications on Pure and Applied Mathematics, vol. 21, no. 5, pp. 467-490, 1968.

[7] C. S. Cardner, J. M. Creence, M. D. Kruskal, and R. M. Miura, "Method for solving the Korteweg-deVries equation," Physical Review Letters, vol. 1, no. 19, pp. 1095-1097, 1967.

[8] R. Hirota, Topics in Current Physics, Springer-Verlag, Berlin. Germany, 1980.

[9] S. J. Liao, The proposed homotopy analysis technique for the solution of nonlinear problems, Ph.D. thesis, Shanghai Jiao Tong University, Shanghai, China, 1992.

[10] S. Liao and Y. Tan, "A general approach to obtain series solutions of nonlinear differential equations," Studies in Applied Mathematics, vol. 119, no. 4, pp. 297-354, 2007.

[11] G. Adomian, Solving Frontier Problems of Physics: The Decomposition Method, Kluwer Academic Publishers, Amsterdam, Netherlands, 1994.

[12] C. Kesan, "Taylor polynomial solutions of linear differential equations," Applied Mathematics and Computation, vol. 142, no. 1, pp. 155-165, 2003.

[13] B. Bülbül and M. Sezer, "Taylor polynomial solution of hyperbolic type partial differential equations with constant coefficients," International Journal of Computer Mathematics, vol. 88, no. 3, pp. 533-544, 2011.

[14] C. P. Filipich, L. T. Villa, and R. O. Grossi, "The power series method in the effectiveness factor calculations," Latin American Applied Research, vol. 40, no. 3, pp. 207-212, 2010.

[15] C. P. Filipich, M. B. Rosales, and F. Buezas, "Some nonlinear mechanical problems solved with analytical solution," Latin American Applied Research, vol. 34, pp. 101-109, 2004.

[16] P. P. Banerjee, "A simplified approach to solving nonlinear dispersive equations using a power series method," Proceedings of the IEEE, vol. 74, no. 9, pp. 1288-1290, 1986.

[17] V. Fairén, V. López, and L. Conde, "Power series approximation to solutions of nonlinear systems of differential equations," American Journal of Physics, vol. 56, no. 1, pp. 57-61, 1988.

[18] A. S. Nuseir, Al-Hasson, and Abeer, "Power series solution for nonlinear system of partial differential equations," Applied Mathematical Sciences, vol. 6, no. 104, pp. 5147-5159, 2012.
[19] H. Quevedo, "General static axisymmetric solution of Einstein's vacuum field equations in prolate spheroidal coordinates," Physical Review D, vol. 39, no. 10, pp. 2904-2911, 1989.

[20] J.-S. Chen, C.-F. Ni, and C.-P. Liang, "Analytical power series solutions to the two-dimensional advection-dispersion equation with distance-dependent dispersivities," Hydrological Processes, vol. 22, no. 24, pp. 4670-4678, 2008.

[21] A. D. Polyanin and V. F. Zaitsev, Handbook of Nonlinear Partial Differential Equations, Chapman \& Hall/CRC, Boca Raton-London, 1986.

[22] D. Baleanu, K. Diethelm, E. Scalas, and J. J. Trujillo, "Fractional calculus: models and numerical methods of series on complexity, nonlinearity, and chaos. world scientific," 2012.

[23] M. A. Matlob and Y. Jamali, "The concepts and applications of fractional order differential calculus in modeling of viscoelastic systems: a primer," Critical Reviews in Biomedical Engineering, vol. 47, no. 4, pp. 249-276, 2019.

[24] V. E. Tarasov, Fractional Dynamics: Applications of Fractional Calculus to Dynamics of Particles, Fields, and Media, Springer, Berlin, Germany, 2011.

[25] V. E. Tarasov, "On the history of mathematical economics: application of fractional calculus," Mathematics, vol. 7, no. 509, pp. 1-28, 2019.

[26] C. N. Angstmann, A. M. Erickson, B. I. Henry, A. V. McGann, J. M. Murray, and J. A. Nichols, "Fractional order compartment models," SIAM Journal on Applied Mathematics, vol. 77, no. 2, pp. 430-446, 2017.

[27] D. A. Benson, S. W. Wheatcraft, and M. M. Meerschaert, "Application of a fractional advection-dispersion equation," Water Resources Research, vol. 36, no. 6, pp. 1403-1412, 2000.

[28] C. N. Angstmann, B. I. Henry, and A. V. McGann, "A fractional order recovery SIR model from a stochastic process," Bulletin of Mathematical Biology, vol. 78, no. 3, pp. 468-499, 2016.

[29] B. I. Henry, T. A. M. Langlands, and S. L. Wearne, "Fractional cable models for spiny neuronal dendrites," Physical Review Letters, vol. 100, no. 12, pp. 1-4, Article ID 128103, 2008.

[30] R. Metzler and J. Klafter, "The random walk's guide to anomalous diffusion: a fractional dynamics approach," Physics Reports, vol. 339, no. 1, pp. 1-77, 2000.

[31] I. Podlubny, Fractional Differential Equations of Mathematics in Science and Engineering, Academic Press, New York, NY, USA, 1998.

[32] S. Kazem, "Exact solution of some linear fractional differential equations by Laplace transform," International Journal of Nonlinear Science, vol. 16, pp. 3-11, 2013.

[33] S.-D. Lin and C.-H. Lu, "Laplace transforms for solving some families of fractional differential equations and their applications," Advances in Difference Equations, vol. 137, pp. 1-9, 2013.

[34] M. Rivero, L. Rodríguez-Germá, and J. J. Trujillo, "Linear fractional differential equations with variable coefficients," Applied Mathematics Letters, vol. 21, no. 9, pp. 892-897, 2008.

[35] M.-F. Li, J.-R. Ren, and T. Zhu, "Series expansion in fractional calculus and fractional differential equations," arXiv: 0910.4819V2, 2009.

[36] A. El-Ajou, O. Arqub, Z. Zhour, and S. Momani, "New results on fractional power series: theories and applications," Entropy, vol. 15, no. 12, pp. 5305-5323, 2013.

[37] M. Al-Refai, M. Ali Hajji, and M. I. Syam, "An efficient series solution for fractional differential equations," Abstract and Applied Analysis, vol. 2014, Article ID 891837, 9 pages, 2014.

[38] F. Xu, Y. Gao, X. Yang, and H. Zhang, "Construction of fractional power series solutions to fractional Boussinesq 
equations using residual power series method," Mathematical Problems in Engineering, vol. 2016, Article ID 5492535, 15 pages, 2016.

[39] M. K. Al-Srihin and M. Al-Refai, "An efficient series solution for nonlinear multiterm fractional differential equations," Discrete Dynamics in Nature and Society, vol. 2016, Article ID 5234151, 11 pages, 2017.

[40] F. Ren and Y. Hu, "The fractional power series method an efficient candidate for solving fractional systems," Thermal Science, vol. 22, no. 4, pp. 1745-1751, 2018.

[41] M. Alquran, I. Jaradat, and S. Sivasundaram, "Elegant scheme for solving Caputo-time-fractional integrodifferential equations," Nonlinear Studies, vol. 25, pp. 385-393, 2018.

[42] R. Shat, S. Al-Refai, I. Alhamayda, A. Sharan, and M. Al-Refai, "The fractional Laguerre equation: series solutions and fractional Laguerre functions," Frontiers in Applied Mathematics and Statistics, vol. 5, pp. 1-6, 2019.

[43] A. Freihet, S. Hasan, M. Al-Smadi, M. Gaith, and S. Momani, "Construction of fractional power series solutions to the fractional stiff system using residual functions algorithm," Advances in Difference Equations, vol. 95, 2019.

[44] H. Habenom, D. 1. Suthar, and M. Aychluh, "Solution of fractional Fokker Planck equation using fractional power series method," Journal of Science and Arts, vol. 3, no. 48, pp. 593-600, 2019.

[45] E. Lopez-Sandoval, A. Mello, and J. J. Godina-Nava, "Power series solution to non-linear partial differential equations of mathematical physics," 2016, https://arxiv.org/ftp/arxiv/ papers/1206/1206.2346.pdf.

[46] E. Lopez-Sandoval, A. Mello, and J. J. Godina-Nava, "Power series solution to non-linear partial differential equations: accuracy and simplicity," Transaction on Mathematics, vol. 2, no. 3, pp. 59-80, 2016.

[47] E. Lopez-Sandoval, A. Mello, J. J. Godina-Nava, and A. R. Samana, "Power series solution for nonlinear burgerstype equations," Abstract And Applied Analysis, vol. 2015, Article ID 712584, 9 pages, 2015.

[48] C. N. Angstmann and B. I. Henry, "Generalized fractional power series solutions for fractional differential equations," Applied Mathematics Letters, vol. 102, Article ID 106107, 2020.

[49] M. Aylin Bayrak and A. Demir, "A new approach for spacetime fractional partial differential equations by Residual power series method," Applied Mathematics and Computation, vol. 336, pp. 215-230, 2018.

[50] A. I. Ali and M. I. Bhatti, "Comparison of Aboodh transformation and differential transformation method numerically," Science International Lahore (Pakistan), vol. 27, no. 2, pp. 873-879, 2015. 